\title{
Genetics and oncology
}

\section{Gene expression and antibodies for diagnostics.}

\section{MACS Anti-Melanoma MicroBeads \\ Miltenyi Biotec www.miltenyibiotec.com \\ Combined cell enrichment and staining}

Anti-Melanoma MicroBeads are used to enrich disseminated melanoma cells from peripheral blood, bone marrow and lymphoid tissue and for purifying melanoma cells from single-cell suspensions of skin biopsies. The antibody, clone 9.2.27, conjugated to MicroBeads, recognizes a surface antigen expressed by most melanomas. Following enrichment, melanoma cells can be analysed by immunocytochemistry, flow cytometry or RT-PCR, or taken directly into culture.

Reader Service No. 100

\section{GeneMap}

Genomic Solutions www.genomicsolutions.com Pre-printed microarrays

The GeneMap array consists of 1,152 cancerrelated genes spotted in duplicate within a $9 \times 9,32$-patch array, with a centre-to-centre spacing between spots set at $400 \mu \mathrm{m}$. A control gene, the $Q$ gene from lambda, is spotted at the Al position of each patch, and there are eight blanks within each patch. A gene list can be downloaded from the company's website.

Reader Service No. 101

\section{c-Met}

Zymed www.zymed.com

Prognostic marker for cancers

Rabbit anti-c-Met antibody (PAD: CVD13) can be used in immunohistochemistry applications for the detection of formalin-fixed, paraffin-embedded tissues and western blotting of human samples. c-Met (HGFr) is a proto-oncogene (locus 7q21q31) and a cellsurface receptor for hepatocyte growth factor. c-Met is overexpressed in many cancers and often amplified between tumorigenesis and metastasis. As such, it may be a useful prognostic marker in breast cancer, gastric carcinoma, endometrial carcinoma and others.

Reader Service No. 102

GeneSystem320

KPL www.kpl.com

A fragmented approach

This system uses a defined set of 320 PCR primers and dedicated software available over the Internet to identify known genes or for gene discovery. A message fragmentation protocol involves isolation of actively transcribing genes, isolating a single fragment per mRNA molecule, and conducting specific combinatorial oligo PCR on the fragments. GS320 is available for human, mouse and rat. Reader Service No. 103

\section{WAVE for ATM}

Transgenomic www.transgenomic.co.uk Detecting ATM exon 42 mutations

The Transgenomic WAVE Nucleic Acid Fragment Analysis System performs rapid, automated separation and quantification of singleand double-stranded nucleic-acid fragments, allowing for high throughput, greater accuracy, higher resolution, and substantial savings in cost per sample. The company's Application Note 108 covers the use of WAVE technology for the detection of the ATM (ataxia-telangiectasia) gene.

Reader Service No. 104

\section{Beclin 1 antibody}

Novus Biologicals www.novus-biologicals.com Antibody to key autophagy protein

Novus have introduced an antibody to beclin 1. beclin 1 is the first identified mammalian gene to mediate autophagy, and has also been attributed with tumour suppression and antiviral functions. It is speculated that Beclin 1 may work through induction of autophagy to negatively regulate tumorigenesis and controlviral infections.

Reader Service No. 105

\section{GeneXPRO contract services}

InnoGenex www.innogenex.com Gene profiling at your service

GeneXPRO will carry out assays to analyse cellular and tissue-level expression for gene expression profiling and perform target localization and validation. Gene profiling services include design, synthesis and labelling of probes, correlation of gene expression profile

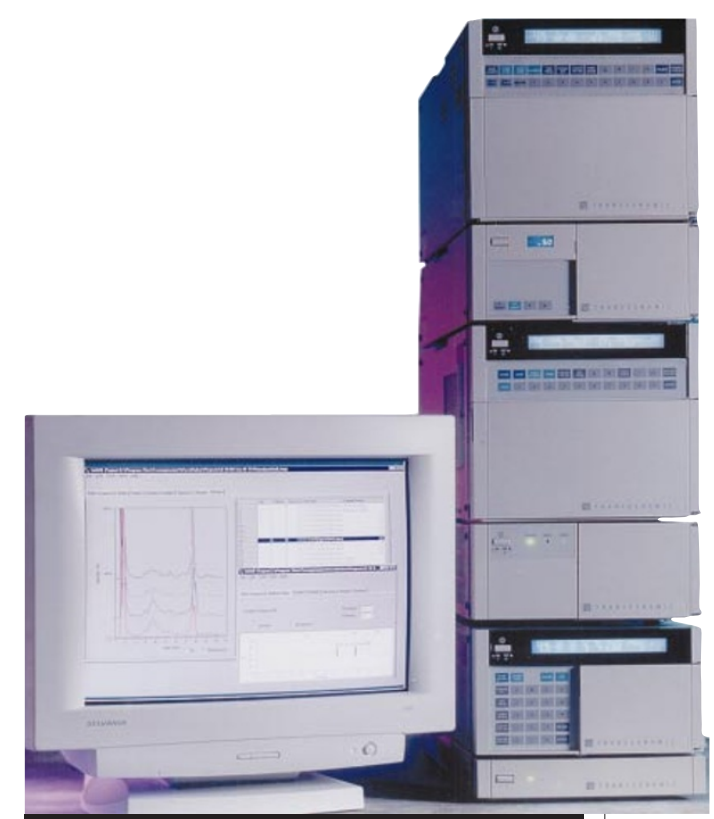

Catch a WAVE.

with normal and pathogenic conditions on cells, tissues and tissue arrays, and determination of changes in gene expression in response to candidate drugs. Other services available include screening mouse monoclonal antibodies on mouse tissues, immunohistochemistry and in situ hybridization on human and rodent tissues.

Reader Service No. 106

COLARIS

Myriad www.myriad.com

Calculated risk

This predictive test assesses an individual's risk of developing hereditary colon cancer based on the presence of a mutation in either of two genes. The same mutations also substantially increase a woman's risk of endometrial cancer. A positive COLARIS test result indicates an $80 \%$ lifetime risk of colon cancer, and a $40-60 \%$ risk of uterine cancer in women.

Reader Service No. 107

These notes are compiled in the Nature office

from information provided by the manufacturers.

ADVERTISEMENTS

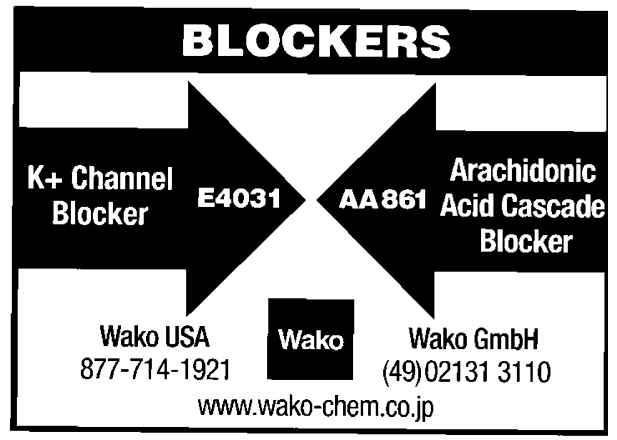

READER ENQUIRY NO. 35

\section{$\mathrm{BACs} \cdot \mathrm{YACs} \cdot \mathrm{PACs}$ cDNAs \\ Research Genetics provides clones and colony membranes for a number of human, mouse and rat libraries as well as a custom screening service for the libraries. \\ Research Genetics \\ U.S. or Canada 800-533-4363 U.K. 0-800-89-1393 FAX 256-536-9016 \\ Check out our homepage at http://www.resgen.com}

READER ENQUIRY NO. 6 